\title{
Model Based Fusion of Laser and Camera: Range Discontinuities and Motion Consistency
}

\author{
Jonas Nygårds \\ Robotics /Autonomous Mechanical Systems \\ Linköping University \\ S-58183 Linköping, Sweden. \\ jonny@ikp.liu.se \\ and \\ Swedish Defence Research Establishment \\ P.O.Box 1165, SE-581 11 Linkoping
}

\author{
Åke Wernersson \\ Robotics \& Automation \\ Luleå University of Technology \\ S-97187 Luleå, Sweden. \\ akewe@sm.luth.se \\ and
}

Swedish Defence Research Establishment

P.O.Box 1165, SE-581 11 Linkoping

\begin{abstract}
Consider a robot to measure or operate on man made objects randomly located in the workspace. The optronic sensing onboard the robot are a scanning range measuring time-of-flight laser and a CCD camera. The plane surfaces are modeled and parmeters extracted using the Radon/Hough transform. This extraction is very robust and motion is also included in a natural way. This paper gives additional results for range discontinuities. A multiple model framework for fusion of sensor information from laser and camera using parametric models of planar and cylindrical surfaces is suggested. An important issue is the mutual consistency between the motion, the range discontinuitym, occlusion and properties of the sensor combination.

Typical applications are; Robust features for use during navigation in cluttered areas. Models for verification and updating of CAD-models when navigating inside buildings and industrial plants. Accumulating sensor readings into a map during operation of a telecommanded robot.
\end{abstract}

Keywords: multi-sensor-fusion, optronic sensors, range laser (LIDAR), camera (CCD), Radon / Hough transform, data association, estimation, sub-voxel laser measurement model.

\section{Introduction}

The present paper considers model-based fusion using the interacting-multiple-model approach (IMM [BaS93]). Special focus is on modeling of planar surfaces.

Related work on navigation of mobile robots based on plane surfaces are found in, say; [Cro89], [HBC96], [LFW96], [MoC89] and [SSC90] while [KLO93], presents a non parametric solution for natural environments. Special references to covariances for estimating planes are found in [Kan94] and [Lar95].

The result complement earlier results on stochastic maps [MoC89], [SSC90] and related work as [Tar92] towards an important class of applications. The motivation behind the work is the need for explicit models in design of feedback control and sensor planning as in [NNW97] and [NiN96]. Most work today is concerned with calibration of LIDAR and camera followed by texture mapping of camera intensity on lidar $21 / 2 \mathrm{D}$ model or triangulated meshes. [GRB94], [GSBL94], [HSIW96] and [HB3R97],

\section{Laser Measurement model}

First consider the laser measurements.

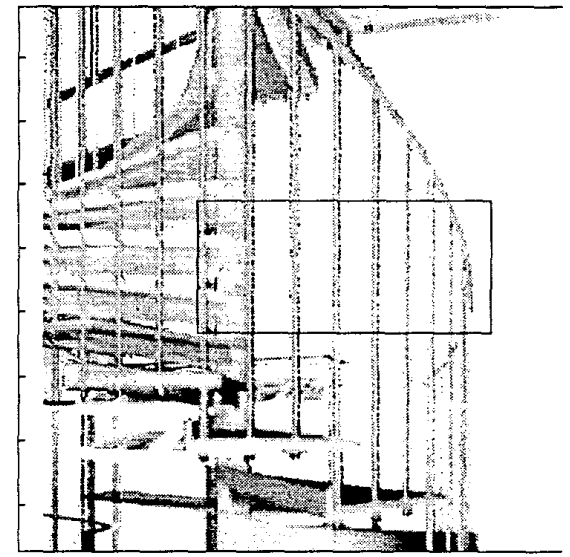

Figure 1 The image illustrates the returned amplitude for a laser scan. The square on the railing in the middle represents the measurements that are selected to show the effect the laser lobe width in the plots below.

In Figure 1 the detected laser amplitude of a scan is displayed as an image. The scan is from an indoor scene with a spiral staircase in front of a wall with a bookshelf against the wall to the left. An almost empty bookshelf is behind the spiral staircase and just in front of the wall at 


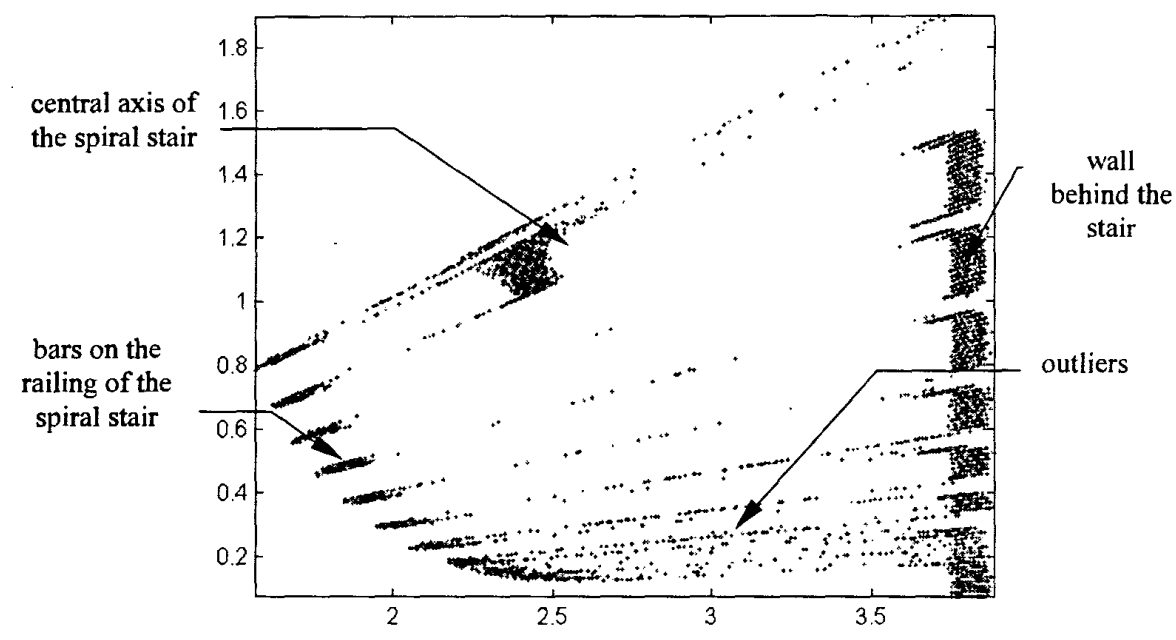

Figure 2 Part from measurements of a staircase. Note the jump-edge smearing when the laser lobe is both on the railing of the stair as well as on the wall in the background.

3.4 meter. Notice that the two upper shelves are inclined (for magazines) while the lower shelves are horizontal. In the image a subset of measurements on the railing of the stair are marked by a rectangle. The selected measurements on the railing are plotted in Figure 2, using a top view so that the false measurements caused by the lobe width are apparent. The false measurements are found as unevenly distributed points along lines between the railing and the wall behind.

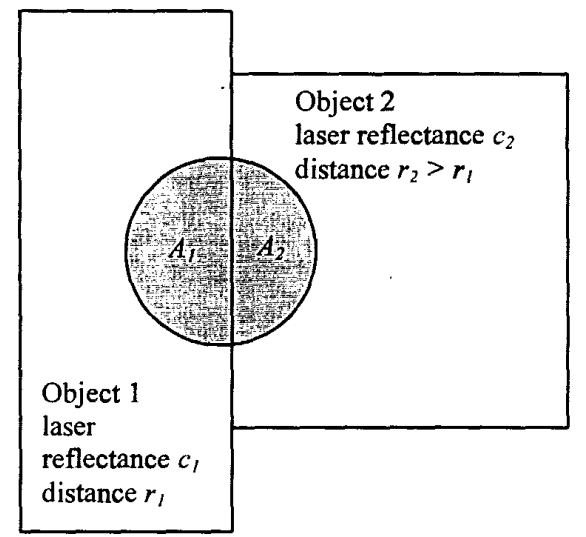

Figure 3 The case where the laser beam is split into echoes at two distances.In Figure 4 the laser beam is sweeping over an aluminium profile with a gradually larger part of the beam being reflected from the background.

In this experiment the range measurements from the laser gradually move from the closer aluminium profile at 4.65 $\mathrm{m}$. to the background at $4.98 \mathrm{~m}$. Some measurements falls between the two objects causing false ranges. The camera is mounted approximately $0,2 \mathrm{~m}$ below the laser.
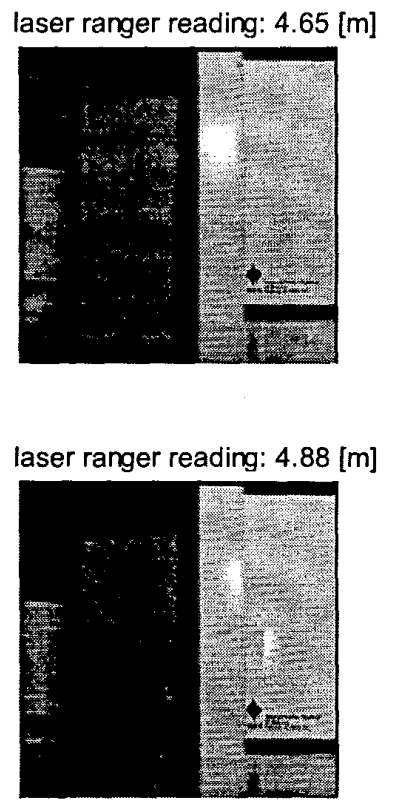

Figure 4 The images illustrates the effect of partial illumination of two objects by the range measuring laser. If the objects are closer to each other than one pulse length the range reading becomes a weighted mean between the two distances with the weight depending on the magnitude of the reflection from each object.

By increasing the separation between the aluminium profile and the background, the false measurements disappear when the distance between the two objects 
exceeds $2.6[\mathrm{~m}]$. The interpretation is that the two objects now are separated more than one pulse length. This behaviour, causing outliers at edges prompted early laser/vision fusion architectures to use vision to find edges where the laser measurements were [Nyg98]. By modelling this behaviour instead the laser can be used to detect occluding edges with "sub-voxel" resolution.

When two objects are within one laser pulse as in Figure 3 and Figure 4 the following sub-voxel range-measurement model is suggested.

$$
r=w_{1} r_{1}+w_{2} r_{2}+v \quad v \in N\left(0, \sigma_{\text {jump }}^{2}\right)
$$

where

$$
\sigma_{\text {jump }}^{2} \gg \sigma_{r}^{2}
$$

and

$$
w_{1}=\frac{A_{1} c_{1}}{A_{1} c_{1}+A_{2} c_{2}} \quad w_{2}=\frac{A_{2} c_{2}}{A_{1} c_{1}+A_{2} c_{2}} \text { (2) }
$$

The model in (1) is does not fully model the interplay between the laser pulse and the detector but it captures the main properties.

However with a single scan of measurements from only one viewpoint this case cannot be discerned from a case where the two objects are connected with a plane.

\section{Stochastic Geometric Models}

Measurements on a plane as in Figure 5 are modelled by: $r(\alpha, \beta, u)=\rho /(\cos \alpha \cos \beta \cos \phi \cos \theta+$

$$
\begin{aligned}
& \sin \alpha \cos \beta \sin \phi \cos \theta-\sin \beta \sin \theta)+v \\
& v \in N\left(0, \sigma_{r}^{2}\right) \quad \sigma_{r}^{2}<\sigma_{\text {jump }}^{2}
\end{aligned}
$$
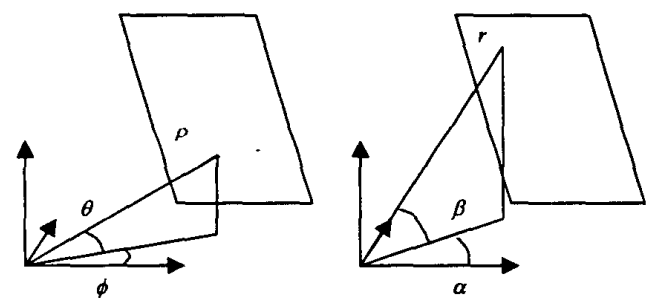

Figure 5 the parameters for measurements of a plane. To the left the parameters of the plane where $\rho$ is the perpendicular distance to the plane and $\theta, \phi$ the orientations of the perpendicular. To the right the spherical coordinates for one measurement on the plane are illustrated.

Measurements that lie on edges i. e. where the difference in distance between neighbouring measurements are larger than measurement uncertainty belong to one of two models:

1. An occlusion jump edge as in Figure 6 with measurement model (2)

2. A plane connecting object 1 and 2 measurement model (3)

Now, consider edges detected in a concurrent camera image. Fusing these edges with the plane models from the laser we get three possible measurement models:
1. The edge is caused by texture and lies in one of the laser surfaces

2. The edge is caused by the intersection of two surfaces. 3. The edge belongs to an occlusion jump edge.

The first two cases result in a line of intersection, while the third case gives a plane of occlusion. In all three cases the information of the camera edges are modeled as planes through the camera optical center and through the edgepoints detected on the image-plane. For cylindrical edges the method presented in [SzW98] will be applied.

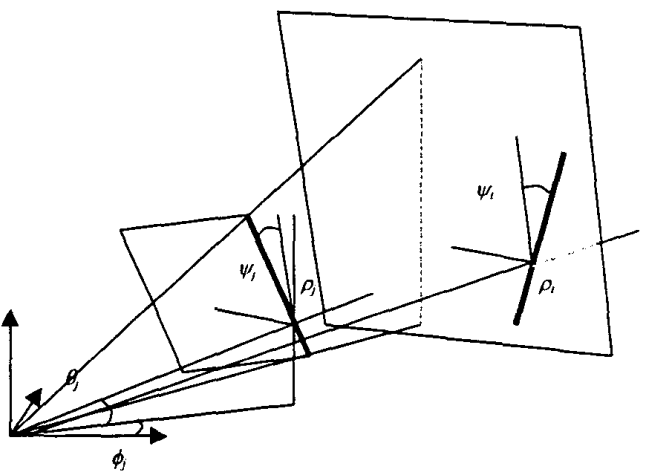

Figure 6 the parameters for a jump and texture edges (subscripted $j / t$ ). The perpendicular distance $\rho$ with orientation $\theta, \phi$ and the rotation around the perpendicular $\psi$ (measured from the $z$-axis in a $x-y-z$ coordinate system with $y$ horizontal and $x$ parallel with the perpendicular)

An approximation of the information from LIDAR measurements of a planar surface is given in [NW98].

For 3D measurements $z_{i}=\left[x_{i}, y_{i}, z_{i}\right]+\varepsilon_{i}$ the plane is parmetrised by $u=[\rho, \theta, \phi]$. Introduce the distance function:

$$
\begin{aligned}
& F(\mathbf{z}, \mathbf{u})= \\
& x \cos (\phi) \cos (\theta)+y \sin (\phi) \cos (\theta)-z \sin (\theta)-\rho=0
\end{aligned}
$$

where the noise is modelled as $E\left[\varepsilon_{i}\right]=0 \quad E\left[\varepsilon_{i} \varepsilon_{i}^{T}\right]=R$

The information matrix from a single measurement is then approximated to the first order by the matrix

$$
\frac{\partial F(\mathbf{z}, \mathbf{u})}{\partial \mathbf{u}}\left(\frac{\partial F(\mathbf{z}, \mathbf{u})}{\partial \mathbf{z}} \operatorname{cov}(\mathbf{z})\left(\frac{\partial F(\mathbf{z}, \mathbf{u})}{\partial \mathbf{z}}\right)^{T}\right)^{-1}\left(\frac{\partial F(\mathbf{z}, \mathbf{u})}{\partial \mathbf{u}}\right)^{T}
$$

The corresponding information turn out to be

$$
\sum_{i=1}^{N} \frac{1}{\sigma_{f f}^{2}}\left[\begin{array}{ccc}
1 & \xi_{i} & -\eta_{i} \cos (\theta) \\
\xi_{i} & \xi_{i}^{2} & -\xi_{i} \eta_{i} \cos (\theta) \\
-\eta_{i} \cos (\theta) & -\xi_{i} \eta_{i} \cos (\theta) & \eta_{i}^{2} \cos ^{2}(\theta)
\end{array}\right] \text { (5) }
$$

where the $(\eta, \xi)$ parameterisation is

$$
\eta=-x \sin (\phi)+y \cos (\phi)
$$

$\xi=x \cos (\phi) \sin (\theta)+y \sin (\phi) \sin (\theta)+z \cos (\theta)$

and the projected noise is

$$
\begin{aligned}
\sigma_{f}^{2}= & {\left[\begin{array}{lll}
\cos (\phi) \cos (\theta) & \sin (\phi) \cos (\theta) & -\sin (\theta)
\end{array}\right] * } \\
& R\left[\begin{array}{lll}
\cos (\phi) \cos (\theta) & \sin (\phi) \cos (\theta) & -\sin (\theta)
\end{array}\right]^{T}
\end{aligned}
$$


For the laser measurements the inverse of the information model (5) gives an approximation of the covariance for a plane-estimate based on $m x n$ measurements with interpoint distance $\Delta$ as:

$$
\operatorname{cov}\left(\left[\begin{array}{lll}
\rho & \theta & \phi
\end{array}\right]\right)=\left[\begin{array}{ccc}
\sigma_{c}^{2}+\sigma_{\theta}^{2} \eta^{2}+\sigma_{\theta}^{2} \xi^{2} & -\xi \sigma_{\theta}^{2} & \eta \sigma^{2} \\
-\xi \sigma_{\theta}^{2} & \sigma_{\theta}^{2} & 0 \\
\eta \sigma_{*}^{2} & 0 & \sigma_{\bullet}^{2}
\end{array}\right]
$$$$
\text { where } \sigma_{c}^{2}=\frac{\sigma_{r}^{2}}{m n}
$$

$$
\sigma_{\theta}^{2}=\frac{12 \sigma_{c}^{2}}{\Delta^{2}\left(n^{2}-1\right)} \quad \sigma_{\theta}^{2}=\frac{12 \sigma_{c}^{2}}{\Delta^{2}\left(m^{2}-1\right)}
$$

The same model can be used for the camera edge using virtual measurements on a virtuel image plane in front of the camera to get a plane going through the optical center giving $2 \times n$ measurments:

$$
\left[\begin{array}{lll}
x_{1} & \cdots & x_{n} \\
y_{1} & \cdots & y_{n} \\
f & \cdots & f \\
-x_{1} & \cdots & -x_{n} \\
-y_{1} & \cdots & -y_{n} \\
-f & \cdots & -f
\end{array}\right]
$$

The fusion of camera and laser measurments gives an updated line of intersection. In a coordinate system oriented with $\mathrm{x}$-axis along the normal of plane 1 and $z$-axis along the line of intersection, the estimate of the $x-y$ coordinates for the intersection has covariance matrix:

$$
\begin{aligned}
& C\left(x_{i}\right)=\left(C\left(x_{1 i}\right)^{-1}+C\left(x_{2 i}\right)^{-1}\right) \\
& =\left[\begin{array}{cc}
\sigma_{a}^{2} & \sigma_{a}^{2} \cot \left(\phi_{2}\right) \\
\sigma_{a}^{2} \cot \left(\phi_{2}\right) & \frac{\sigma_{a}^{2} \cos ^{2}\left(\phi_{2}\right)+\sigma_{b}^{2}}{\sin ^{2}\left(\phi_{2}\right)}
\end{array}\right]
\end{aligned}
$$

where

$$
\begin{gathered}
\sigma_{a}^{2}=\frac{\sigma_{r}^{2}}{m_{1} n_{1}}\left(1+\eta_{1}^{2} \frac{12}{\Delta^{2}\left(m_{1}^{2}-1\right)}+\xi_{1}^{2} \frac{12}{\Delta^{2}\left(n_{1}^{2}-1\right)}\right) \\
\sigma_{b}^{2}=\frac{\sigma_{r}^{2}}{2 n_{2}}\left(1+\eta_{2}^{2} \frac{12}{f^{2}}+\xi_{2}^{2} \frac{12}{\left(n_{2}^{2}-1\right)}\right) \\
\operatorname{cov}\left(n_{1}\right)=\left[\begin{array}{ccc}
0 & 0 & 0 \\
0 & \sigma_{01}^{2} & 0 \\
0 & 0 & \sigma_{\theta 1}^{2}
\end{array}\right]
\end{gathered}
$$

where $\sigma_{c 1}^{2}=\frac{\sigma_{r}^{2}}{m_{1} n_{1}}$

$$
\sigma_{\theta 1}^{2}=\frac{12 \sigma_{c 1}^{2}}{\Delta^{2}\left(n_{1}{ }^{2}-1\right)} \quad \sigma_{\phi 1}^{2}=\frac{12 \sigma_{c 1}^{2}}{\Delta^{2}\left(m_{1}{ }^{2}-1\right)}
$$

$\operatorname{cov}\left(n_{2}\right)=$

$$
\left[\begin{array}{ccc}
\sin \left(\phi_{2}\right)^{2} \sigma_{\phi 2}^{2} & -\sin \left(\phi_{2}\right) \cos \left(\phi_{2}\right) \sigma_{\phi 2}^{2} & 0 \\
-\sin \left(\phi_{2}\right) \cos \left(\phi_{2}\right) \sigma_{\phi 2}^{2} & \cos \left(\phi_{2}\right)^{2} \sigma_{\phi 2}^{2} & 0 \\
0 & 0 & \sigma_{\theta 2}^{2}
\end{array}\right]
$$

where $\sigma_{c 2}^{2}=\frac{\sigma_{r}^{2}}{2 n_{2}}$

$$
\sigma_{\theta 2}^{2}=\frac{12 \sigma_{c 2}^{2}}{\left(n_{2}^{2}-1\right)} \quad \sigma_{\phi 2}^{2}=\frac{12 \sigma_{c 2}^{2}}{f^{2}}
$$

Giving the covariance for the direction of the intersection

$$
\begin{aligned}
& C\left(\left(\hat{\boldsymbol{n}}_{1} \times \hat{\boldsymbol{n}}_{2}\right) /\left\|\hat{\boldsymbol{n}}_{1} \times \hat{\boldsymbol{n}}_{2}\right\|\right)= \\
& \frac{\left(I-\boldsymbol{e}_{z} \boldsymbol{e}_{z}^{T}\right)\left(\tilde{\boldsymbol{n}}_{2} C\left(\hat{\boldsymbol{n}}_{1}\right) \tilde{\boldsymbol{n}}_{2}+\tilde{\boldsymbol{n}}_{1} C\left(\hat{\boldsymbol{n}}_{2}\right) \tilde{\boldsymbol{n}}_{1}\right)\left(I-\boldsymbol{e}_{2} \boldsymbol{e}_{z}^{T}\right)}{\sin \left(\phi_{2}\right)^{2}}
\end{aligned}
$$

giving

$$
C\left(e_{2}\right)=\left[\begin{array}{ccc}
\sigma_{\theta 1} & -\frac{\sigma_{\theta 1} \cos \left(\phi_{2}\right)}{\sin \left(\phi_{2}\right)} & 0 \\
-\frac{\sigma_{\theta 1} \cos \left(\phi_{2}\right)}{\sin \left(\phi_{2}\right)} & \frac{\sigma_{\theta 2}+\sigma_{\theta 1} \cos \left(\phi_{2}\right)^{2}}{\sin \left(\phi_{2}\right)^{2}} & 0 \\
0 & 0 & 0
\end{array}\right](14)
$$

\section{Multiple model framework}

Given an initial laser scan with segmentation into surface patches, the model parameters are estimated. The parameters are:

- For plane surface $\mathbf{u}=[\rho, \theta, \phi]$

- For cylinder $\mathrm{u}=[\rho, \theta, \phi, r, \psi]$

- Connecting edges between neighbouring planes $\mathrm{u}=[\rho, \theta, \phi, \psi]$ (derived from the planes intersection)

- Possible jump-edge voxels, two hypothesises occluding plane $\mathrm{u}=[\rho, \theta, \phi]$ occluding edge $\mathbf{u}=[\rho, \theta, \phi, \psi]$

The detected camera edges are then gated against the laser edges giving

- Texture edges $\mathbf{u}=[\rho, \theta, \phi, \psi]$ (derived from intersection with the laser planes )

- connecting edge $\mathrm{u}=[\rho, \theta, \phi, \psi]$ (fused with laser connecting edge)

- occluding edge $\mathbf{u}=[\rho, \theta, \phi, \psi]$ (fused with laser occluded edge)

The multiple models are then pruned during motion. For a possible occluding edge the platform motion makes the occluding plane hypothesis improbable or verifies the occluding plane. 


\section{Experiments}

Jump-edge smearing

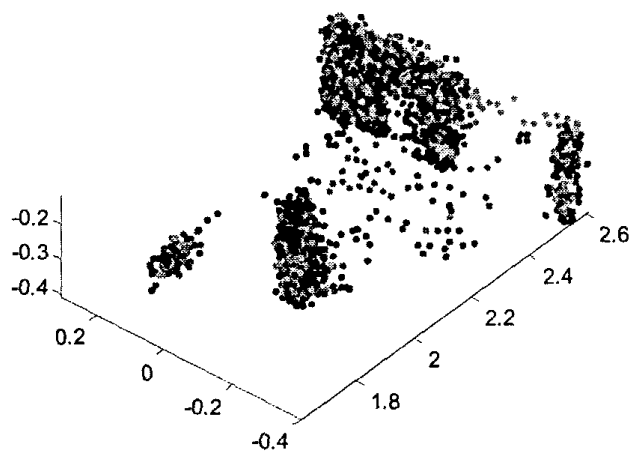

Figure 7 LIDAR measurements (IBEO laser in single shot mode) corresponding to the camera image below. Note the jump edge smearing at the top and right side of the book.

Fusing the camera data

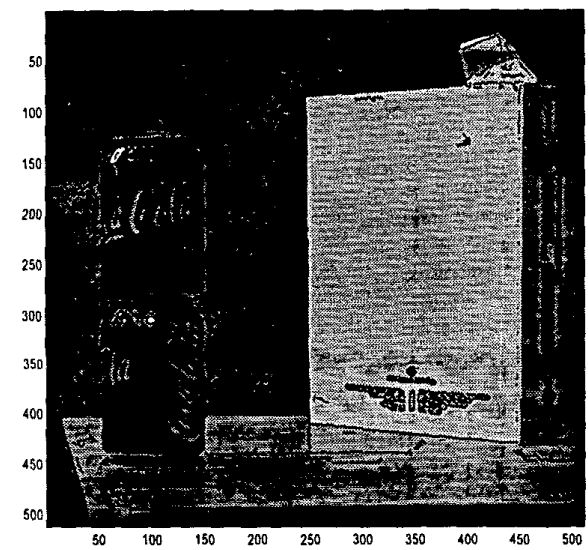

Figure 8 Camera image with detected edges superpositioned.

Additional experiments, color images and animations making jump-edge smearing points more visible can be found on:

URL: http://idefix.ikp.liu.se/rames/laser/FusionExp.html

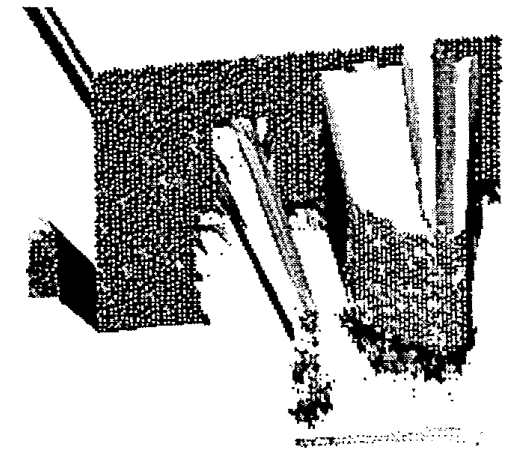

Figure 9 LIDAR measurements (IBEO laser in High res. averaging mode) corresponding to the camera image above. The camera is mounted approximately $0,2 \mathrm{~m}$ below the laser. The measurements are rendered with artificial light from a slightly different viewpoint and each measurement as a white dot.

\section{Conclusions}

Navigation based on stochastic line / surface segments estimated from range data has proven robust and used in many cases of cluttered workspaces. This paper presented model representations of range discontinuities from a multi-sensor fusion viewpoint. Special considerations are that the measurements are from a moving platform. The main contributions are:

- A sub-voxel model used for laser measurements at range discontinuities where the background is within the pulse-length. By modeling the jump-edgesmearing the laser measurements can be used to detect edges with "sub voxel" resolution instead of throwing away these edge measurements as in earlier camera/laser fusion.

- A multiple model filter, considering sub-voxel laser model, CCD-image edges as texture-, surface- or occlusion- edge.

- Motion as the tool to verify the hypothesises of radius, texture-, surface- or occlusion- edge, and laser "sub-voxel" edge vs. physical surface.

In the present work no attention has been paid to the problem of segmentation and association. However, a good representation of uncertainty is essential for good associations. The multiple model framework is a natural tool for association problems using Bayesian all neighbour methods (JPDA-methods [BSL93]).

\section{References}

[AM79] Anderson, B. D. O. and Moore, J. B. Optimal Filtering, Prentice Hall, 1979.

[BSL93] Bar-Shalom, Y. and Li, X. -R. Estimation and Tracking: Principles, Techniques, and Software, Artech House, 1993. 
[Cro89] Crowley, J. (1989). World Modelling and Position Estimation for a Mobile Robot Using Ultrasonic Ranging, IEEE Int. Conf Robotics and Automation, 1989, pp 674-680.

[D-W88] Durrant-Whyte, H.F., Uncertain Geometry, IEEE Journal of Robotics \& Automation Vol 4, 1988

[GRB94] Godin, G., Rioux, M., and Baribeau, R. "3-D registration using range and intensity information.", SPIE Proceedings, Videometrics III, International Symposium on Photonic and Sensors and Controls for Commercial Applications, Boston, MA. October 31 . November 4, Vol.2350. pp. 279-290. 1994.

[GSBL94] Gagnon, H., Soucy, M., Bergevin, R. and Laurendeau, D. "Registration of multiple range views for automatic 3D model building." In Proceedings IEEE Conference on Computer Vision and Pattern Recognition, pp 581-586, 1994.

[HB3R97] El-Hakim, S.F., Boulanger, P., Blais, F., Beraldin, J.-A. and Roth, G., "A Mobile System for Indoors 3-D Mapping and Positioning," Proc. Optical 3-D Measurement Techniques, Sept. 29-Oct. 2, 1997

[HBC96] Hebért, P. Betgé-Brezetz and Chatila, R. Decoupling Odometry And Exteroceptive Perception In Building A Global World Map of a Mobile Robot: The Use of Local Maps, Proc. IEEE Int. Conf Robotics and Automation, 1996, Minnesota, April 1996, pp 757 764

[HSIW96] Hilton,A. , Stoddart,A.J. , Illingworth,J. and Windeatt,T. "Reliable Surface Reconstruction from Multiple Range Images", ECCV 1996.

[Kan94] Kanatani, K. "Statistical analysis of geometric computation" CVGIP: Image Understanding, Vol.59, No.3, pp. 286-306, 1994

[KL093] P. Klöör, P. Lundquist, P. Ohlsson, A. Wernersson, Change Detection ,IFAC Intelligent Autonomous Vehicles, Southampton, May 93, pp 7176

[Lar95] U. Larsson, Mobile Robot Localisation using Electro-OpticalSensors, Lic. thesis, (HLU-TH-L-1995/42-L--SE) Dec. 1995

[LFW96] U. Larsson, J. Forsberg, Å. Wernersson "Mobile Robot Localisation: Integrating Measurements from a Time-of-Flight Laser", IEEE Transactions on Industrial Electronics, special issue on MultiSensor Fusion and Integration for Intelligent Systems, Vol. 43, No. 3, June 1996. pp. 422-432.

[LFW96] U. Larsson, J. Forsberg, §. Wernersson "Mobile Robot Localisation: Integrating Measurements from a Time-of-Flight Laser", IEEE Transactions on Industrial Electronics, special issue on MultiSensor Fusion and Integration for Intelligent Systems, Vol. 43, No. 3, June 1996. pp. 422-432.

[MoC89] Moutarlier, P. and Chatila, R. (1989) An Experimental System for Incremental Environment Modelling by an Autonomous Mobile Robot, 1st Int Symp. on Experimental Robotics, Montreal, 1989, Springer Verlag.
[NiN96] Nilsson B. and Nygårds J. Sensor Motion Planning with Active Uncertainty Reduction: Gripping and Docking Tasks, Proc. IEEE Int. Conf. on Robotics and Automation, Vol. 4 pp. 3026-3033, Minneapolis, April 22-28,1996.

[NNW96] Nilsson, B., Nygårds, J. and Wernersson, $\AA$. On $2 D$ Posture Estimation with Large Initial Orientation Uncertainty and Range Dependant Noise. Int. Conf. on Automation Robotics and Computer Vision, Singapore 1996

[NNW97] Nilsson B., Nygårds J. and Wernersson $\AA$., "On-Range Sensor Feedback for Mobile Robot Docking Within Prescribed Posture Tolerances.", Journal of Robotic Systems Vol. 14 No. 4, pp. 297. 312, John Wiley \& Sons, Inc., 1997.

[Nyg98] Nygårds, J. "On Robot Feedback from Range Sensors: Reliable Control by Active Reduction of Uncertainty and Ambiguities", Linköping Studies in Science and Technology, Thesis No. 528, 1998.

[SSC90] Smith, R., Self, M. and Cheeseman, P. "Estimating Uncertain Spatial Relationships in Robotics," Autonomous Robot Vehicles, SpringerVerlag, pp 167-193. 1990

[SzW98] Szeliski, R., and Weiss, R. "Robust shape recovery from occluding contours using a linear smoother." International Journal of Computer Vision, 28(1):27-44, June 1998

[Tar92] Tardós, J., D. Representing partial and uncertain sensorial information using the theory of symmetries. Proc. IEEE Int. Conf Robotics and Automation, pp1799-1804, Nice, France, 1992 